\title{
Guillain-Barré syndrome variants in Emilia-Romagna, Italy, 1992-3: incidence, clinical features, and prognosis
}

Emilia-Romagna Study Group on Clinical and Epidemiological Problems in Neurology (for members see appendix)

\begin{abstract}
Objectives-To estimate the incidence rate of Guillain-Barré syndrome variants in an unselected population and to describe their clinical features and prognosis. Methods-A two year prospective multicentre study on the incidence and prognosis of Guillain-Barré syndrome was performed in Emilia-Romagna, northern Italy (3 909512 inhabitants). A surveillance system was instituted within the study area, which comprised all the neurological departments, private and public general hospitals, and practising neurologists. The international classification of diseases (ICD) codes 357.XX (any peripheral neuropathy) of hospital discharges were also reviewed.
\end{abstract}

Results-Data were separately analysed for Miller Fisher syndrome and other Guillain-Barré syndrome variants. During the study period 18 patients with Guillain-Barré syndrome variants including seven with Miller Fisher syndrome were recruited; the incidence rates were $0.14 / 100000 /$ year $(95 \%$ confidence interval (95\% CI) 0.07-0.25) for Guillain-Barré syndrome variants (excluding Miller Fisher syndrome) and $0.09 / 100$ 000/year (95\% CI 0.04-0.18) for Miller Fisher syndrome. Guillain-Barré syndrome variants alone (excluding Miller Fisher syndrome) accounted for $10.5 \%$ of total cases. Death and relapses were not found. Details of clinical, electrophysiological, and CSF findings of Guillain-Barré syndrome variants are provided.

Conclusions-Guillain-Barré syndrome variants other than Miller Fisher syndrome, as obtained through a population based study, account for about $10 \%$ of total cases of Guillain-Barré syndrome and, as a whole, have a good prognosis. Their clinical features are heterogeneous; bifacial weakness (associated with other signs, mainly sensory disturbances) represents the most frequent finding.

(F Neurol Neurosurg Psychiatry 1998;65:218-224)

Dr R D'Alessandro,

Laboratorio di

Neuroepidemiologia

Instituto di Clinica

Neurologica, Dell’Università

di Bologna, Via Ugo Foscolo

7 (Porta Saragozza, 40123

Bologna, Italy.

Received 24 February 1997 and in final revised form 15 January 1998

Accepted 23 January 1998
The clinical boundaries of variants of GuillainBarré syndrome remain undefined. The diagnostic criteria suggested by the ad hoc committee of the National Institute of
Keywords: Guillain-Barré syndrome; variants; incidence
Neurological and Communicative Disorders and Stroke (NINCDS) ${ }^{1-3}$ proved useful for research purposes, but many authorities consider these criteria too restrictive, as the clinical variants are cut off. ${ }^{4}$ Clinical variants of Guillain-Barré syndrome have been described from clinical series, which may be subjected to selection biases. Well recognised variants are Miller Fisher syndrome, pharyngeal-cervicalbrachial, paraparetic, and pure motor variants, sensory loss and areflexia, polyneuritis cranialis, and pure pandysautonomia. ${ }^{245}$ Further regional variants have been described by Ropper. ${ }^{6}$ However, about $6 \%$ of patients with Guillain-Barré syndrome cannot be classified according to the known clinical variants. ${ }^{7}$

This study aimed to describe the epidemiological, clinical, laboratory, and electrodiagnostic features and the prognosis of Guillain-Barré syndrome variants obtained through a population based study.

\section{Methods}

An incidence survey on Guillain-Barré syndrome was performed in the Emilia-Romagna region, Italy (3 909512 inhabitants) from 1 January 1992 to 31 December 1993. Details about the method have been published elsewhere. ${ }^{8}$ Briefly, an alert system was instituted within the study area. This system was based on a network of participating centres which comprised all the neurological units (departments, university clinics, and services) of the Public Health System within the investigation area. During the study period these centres also monitored all the departments (intensive care, rehabilitation, general medicine, geriatrics, and paediatrics) in public and private hospitals to which patients with an acute peripheral nerve disease could be admitted. International classification of diseases (ICD) codes 357.XX (any peripheral neuropathy) of hospital discharges were also reviewed. The residents within the investigation area represented the target population.

The inclusion and exclusion criteria proposed by the NINCDS ad hoc committee ${ }^{1}$ were applied for diagnosis of Guillain-Barré syndrome. Following the diagnostic considerations suggested by Asbury ${ }^{2}$ the patients were considered to have Guillain-Barré syndrome variants when presenting with an acute monophasic peripheral nervous system disease followed by an improvement or remission of symptoms, not fulfilling the clinical features of 
Table 1 Mean annual age specific incidence (per 100000) of Guillain-Barré syndrome(GBS) (GBS NINCDS criteria, Miller Fisher syndrome (MFS), and other GBS variants), for both sexes

\begin{tabular}{|c|c|c|c|c|c|c|c|}
\hline \multirow[b]{2}{*}{$\operatorname{Age}(y)$} & \multirow[b]{2}{*}{ Population (both sexes) } & \multicolumn{2}{|c|}{ GBS NINCDS criteria } & \multicolumn{2}{|c|}{$M F S$} & \multicolumn{2}{|c|}{ Other GBS variants } \\
\hline & & $n$ & Incidence $(95 \% \mathrm{CI})$ & $n$ & Incidence $(95 \% \mathrm{CI})$ & $n$ & Incidence $(95 \% \mathrm{CI})$ \\
\hline $0-9$ & 274710 & 4 & 0.73 & 0 & - & 0 & - \\
\hline $10-19$ & 417903 & 2 & 0.24 & 1 & 0.12 & 0 & - \\
\hline $20-29$ & 584363 & 10 & 0.86 & 1 & 0.09 & 2 & 0.17 \\
\hline $30-39$ & 538841 & 9 & 0.84 & 0 & - & 1 & 0.09 \\
\hline $40-49$ & 531317 & 7 & 0.66 & 3 & 0.28 & 1 & 0.09 \\
\hline $50-59$ & 537814 & 12 & 1.12 & 2 & 0.19 & 3 & 0.28 \\
\hline $60-69$ & 511947 & 24 & 2.34 & 0 & - & 3 & 0.29 \\
\hline$\geqslant 70$ & 512617 & 19 & 1.85 & 0 & - & 1 & 0.10 \\
\hline Total & 3909512 & 87 & $1.11(0.89-1.36)$ & 7 & $0.09(0.04-0.18)$ & 11 & $0.14(0.07-0.25)$ \\
\hline
\end{tabular}

Guillain-Barré syndrome or Miller Fisher syndrome, but associated with albuminocytological dissociation and electrodiagnostic features supportive of the diagnosis of Guillain-Barré syndrome. These cases were accepted as Guillain-Barré syndrome variants only after an agreement was reached among the neurologists who joined the study, in the course of collective discussions held during periodical meetings of the research group. Cases were accepted provided that primary causes of neuropathy (such as diabetes and intoxications) were excluded. According to our protocol all cases underwent serum laboratory investigations to exclude systemic, immunological, and neoplastic diseases.

As previously reported ${ }^{8}$ the occurrence of infective antecedents (influenza, upper respiratory, and gastrointestinal tract infections) during the month preceding the onset of GuillainBarré syndrome was investigated by means of a self-administered questionnaire, and the results were not modified by laboratory investigations. A positivity for Campylobacter jejuni infection was defined as the presence of a positive serological result (IgG antibodies) for $C$ jejuni associated with a definite history of a diarrhoeal illness within the three months preceding onset of Guillain-Barré syndrome.

Antibodies to GM1, GD1b, GD1a, GQ1b, and GM2 were detected through enzyme linked immunosorbent assays (ELISAs), using a standardised protocol. ${ }^{9}$ Antibodies to certain gangliosides were considered positive when the mean difference of absorbance at $450 \mathrm{~nm}$ at a titre of 500 was higher than 0.100 ; positive samples were further titrated and the titre expressed as the highest with a difference of absorbance $>0.050$.

\section{Results}

Data were separately analysed for Miller Fisher syndrome and other variants of Guillain-Barré syndrome (these, from here onwards, will be simply referred to as Guillain-Barré syndrome variants). Results concerning Miller Fisher

Table 2 Frequency of clinical infectious antecedents in the month preceding Guillain-Barré syndrome (GBS) onset for the different diagnostic groups (GBS variants and Miller Fisher syndrome (MFS) plus NINCDS criteria)

\begin{tabular}{lll}
\hline $\begin{array}{l}\text { Clinical infectious antecedents in the } \\
\text { month preceding GBS onset }\end{array}$ & $\begin{array}{l}\text { Other GBS } \\
\text { variants } \\
(n=11) n(\%)\end{array}$ & $\begin{array}{l}\text { GBS NINCDS } \\
\text { criteria plus MFS } \\
(n=94) n(\%)\end{array}$ \\
\hline $\begin{array}{l}\text { Upper respiratory tract infections } \\
\text { Influenza }\end{array}$ & $1(9.1)$ & $43(45.7)$ \\
Gastrointestinal tract infections & $1(9.1)$ & $12(12.8)$ \\
\hline
\end{tabular}

syndrome have been previously reported ${ }^{8}$ and will not be considered here. During the study period the participating centres recruited 24 patients not fulfilling NINCDS diagnostic criteria for Guillain-Barré syndrome or Miller Fisher syndrome; among these 24 patients 13 were thereafter excluded, during collective discussions, because of the lack of improvement or worsening during follow up. These cases were actually diagnosed as manifestations of carcinomatosis, systemic autoimmune diseases, and others. Three other patients were excluded because they were non-residents. Overall, 11 patients with a diagnosis of Guillain-Barré syndrome variants were recruited, with an incidence rate of $0.14 / 100000 /$ year (95\% CI $0.07-0.25)$. Table 1 shows the comparative age-specific incidence rates for the three diagnostic categories (NINCDS criteria, Miller Fisher syndrome, and Guillain-Barré syndrome variants). The age specific incidence rates of Guillain-Barré syndrome variants showed a trend towards an increase with advancing age; it is emphasised, however, that this result is based on a few observations.

The men/women ratio for Guillain-Barré syndrome variants was 0.57 , and the mean age at onset was 51.9 (SD 15.8) years $(\mathrm{p}=0.81 v$ pooled Miller Fisher syndrome and cases fulfilling NINCDS criteria; Kruskal-Wallis test for two groups). Table 2 shows the frequency of clinical antecedent events in the month before onset of disease. Upper respiratory tract infections and influenza were reported in $9.1 \%$ of cases each, and gastrointestinal tract infections in $18.2 \%$; the corresponding figures for pooled Miller Fisher syndrome and cases fulfilling NINCDS criteria were $45.7 \%, 12.8 \%$, and $10.6 \%$ respectively.

Table 3 summarises the clinical features of the 11 patients with Guillain-Barré syndrome variants; here we report their detailed case histories.

CASE 1

This 27 year old woman was in the 34th week of pregnancy. She presented paraesthesias (pins and needles, burning) in the extremities of all four limbs, followed 10 days later by difficulty in speech, eating, and eye closure; she also reported diffuse weakness. When first examined she presented facial diplegia, distal hypopallaesthesia, and vasomotor disturbances (blushing and warming) in the limbs. Other 
Table 3 Patient characteristics and summary of clinical findings of Guillain-Barré syndrome(GBS) variants

\begin{tabular}{|c|c|c|c|c|c|c|}
\hline \multicolumn{3}{|c|}{ Patient } & \multirow[b]{2}{*}{ Clinical features } & \multicolumn{2}{|l|}{$C S F$} & \multirow[b]{2}{*}{ Summary of neurophysiological evaluation } \\
\hline No & $\operatorname{Sex}$ & Age & & $\begin{array}{l}\text { Protein } \\
(m g / d l)\end{array}$ & $\begin{array}{l}\text { Days from } \\
\text { onset }\end{array}$ & \\
\hline 1 & $\mathrm{~F}$ & 27 & $\begin{array}{l}\text { Facial diplegia with distal hypopallaesthesia and } \\
\text { paraesthesias }\end{array}$ & 128 & 18 & Axonal (sensory) and demyelinating (motor) \\
\hline 2 & $\mathrm{~F}$ & 47 & $\begin{array}{l}\text { Facial diplegia, dysarthria, perioral paraesthesias, distal } \\
\text { hypopallaesthesia in the legs }\end{array}$ & 150 & 17 & Demyelinating \\
\hline 3 & M & 68 & $\begin{array}{l}\text { Facial diplegia, hypogeusia, low back pain, } \\
\text { hypopallaesthesia in the legs, areflexia, autonomic } \\
\text { dysfunction }\end{array}$ & 49 & 25 & Demyelinating \\
\hline 4 & M & 36 & $\begin{array}{l}\text { Facial diplegia and paraesthesias, distal upper limb } \\
\text { paraesthesias, pelvic girdle pain }\end{array}$ & 177 & 4 & Demyelinating \\
\hline 5 & $\mathrm{~F}$ & 29 & Bifacial weakness with proximal upper limb hyposthenia & 1000 & 5 & Axonal \\
\hline 6 & $\mathrm{~F}$ & 52 & $\begin{array}{l}\text { Asymmetric motor defect, pain, paraesthesias, nystagmus, } \\
\text { areflexia, hypopallaesthesia }\end{array}$ & 64 & 15 & Axonal \\
\hline 7 & M & 53 & Upper limb motor defect with cervical pain & 1420 & 14 & Axonal \\
\hline 8 & M & 62 & Pure sensory defect & 63 & 29 & Demyelinating \\
\hline 9 & $\mathrm{~F}$ & 58 & $\begin{array}{l}\text { Bilateral ptosis, motor-sensory defect, absent ankle } \\
\text { reflexes, hyperactive knee and upper limb reflexes }\end{array}$ & 66 & 15 & Axonal (sensory) and demyelinating (motor) \\
\hline 10 & $\mathrm{~F}$ & 73 & $\begin{array}{l}\text { Motor-sensory defect, abdominal hypaesthesia, sensory } \\
\text { ataxia }\end{array}$ & 2110 & 24 & Axonal \\
\hline 11 & $\mathrm{~F}$ & 65 & $\begin{array}{l}\text { Dysphonia, dysarthria, dysphagia, bifacial weakness, } \\
\text { hyposthenia of cervical and upper limb muscles, brisk } \\
\text { tendon reflexes, bilateral Babinski's sign }\end{array}$ & 51 & 6 & Axonal \\
\hline
\end{tabular}

sensory modalities, power, and reflexes were normal.

Nerve conduction study showed normal motor (right ulnar and right peroneal) and sensory (right ulnar and left sural) conduction velocities. The $\mathrm{F}$ wave of the right peroneal nerve presented an increased latency and a reduced frequency. The right median nerve showed an increased motor distal latency (DL) and $\mathrm{F}$ wave latency, and a reduced sensory action potential (SAP) amplitude with normal conduction velocities. The CSF had no cells and a protein concentration of $128 \mathrm{mg} / \mathrm{dl}$.

She received intravenous immune globulins (IVIg; $0.4 \mathrm{~g} / \mathrm{kg} /$ day for 5 days); improvement of both the sensory and the facial defect began 2 weeks after onset of symptoms. At the last follow up, 18 months later, she still had a slight bifacial weakness with a moderate facial spasm on the right.

CASE 2

This 46 year old woman was chronically treated with antacids for several episodes of gastrointestinal bleeding which had occurred 3 years earlier. One week before admittance she experienced deep pain and muscle contracture in the cervical and dorsolumbar spine, retrosternal and epigastric pain, melena, nausea, and vomiting. These symptoms were followed by paraesthesias around the mouth, difficulties in pursing the lips, blowing out the cheeks, and speech. Symptoms worsened in 2 days.

Neurological examination disclosed dysarthria, especially for the labials. Her face was immobile and expressionless, except for a slight attempt to close the eyes, and Bell's phenomenon was present on both sides. Deep tendon reflexes were weak in the four limbs; distal hypopallaesthesia was found in the legs.

Neurophysiological findings were diagnostic for a demyelinating disease involving both facial nerves and predominating on the right side. Protein concentration in the CSF was 150 $\mathrm{mg} / \mathrm{dl}$, without cells.

Cerebral CT and MRI were normal. Oesophagogastroduodenoscopy showed gastri- tis and chronic duodenitis, whereas an air contrast barium enema was normal.

She was treated with plasma exchange (three sessions). Muscle stretch reflexes returned to normal 6 weeks after onset, and the neurological defects fully recovered one week later.

\section{CASE 3}

Three weeks before admission this 68 year old man presented with lumbosacral pain radiating to the thighs, worse at night. He was admitted for a bilateral but asymmetric (predominating on the left) facial palsy; three days earlier he had experienced diminution of taste. Neurological evaluation also disclosed absence of muscle stretch reflexes in the forearms and mild hypopallaesthesia in the legs; pain sensation was reduced in the left face.

Examination of the CSF disclosed mildly raised proteins $(49 \mathrm{mg} / \mathrm{dl})$ with 4 cells $/ \mathrm{mm}^{3}$. Laboratory analyses also disclosed increased serum concentrations of creatine phosphokinase $(529 \mathrm{IU} / \mathrm{l}), \operatorname{IgG}(2060 \mathrm{mg} / \mathrm{dl})$, and IgA (489 mg/dl).

Results of neurophysiological evaluation were as follows: (1) R1 and R2 responses of blink reflex were absent when stimulating on the left; stimulation on the right evoked normal R1 and ipsilateral R2, whereas the latency of contralateral R2 was markedly increased (47.7 $\mathrm{ms})$; (2) there were reduced motor conduction velocities (MCVs) of left tibial $(34 \mathrm{~m} / \mathrm{s})$ and right peroneal $(38 \mathrm{~m} / \mathrm{s})$ nerves; (3) there was prolonged DL of right ulnar and right peroneal F wave; (4) median and tibial somatosensory evoked potentials (SEPs) on both sides showed reduced conduction in the peripheral tracts with preserved central conduction time.

During the first 5-6 days after admission, the patient complained of interscapular pain migrating to the lumbar tract and to the thighs. Hypertensive fits occurred, which required sublingual nifedipine.

Treatment with IVIg $(0.4 \mathrm{~g} / \mathrm{kg} /$ day for 5 days) was started on the day after admission. 
Improvement occurred within 2 weeks; 6 months later he had fully recovered.

CASE 4

A man aged 36, one week after fever and a scarlet-like exanthem, presented with pain in the buttocks and proximal thighs, which was worse at night. After this, he developed paraesthesias in the hands and right face, cramps of the tongue muscles, and nuchal pain.

There was facial diplegia; the knee reflex was absent on the left and weak on the right. A first neurophysiological evaluation disclosed normal motor DL, MCV, CMAP amplitude, and late response latency in both peroneal nerves; one week later the motor DL and F wave latencies were prolonged and the MCV were reduced. Concentrations of proteins in CSF were $177 \mathrm{mg} / \mathrm{dl}$, with 8 cells $/ \mathrm{mm}^{3}$. A cerebral CT was normal. Improvement began during IVIg treatment $(0.4 \mathrm{~g} / \mathrm{kg} /$ day for 5 days $)$, and full recovery occurred 3 weeks later.

CASE 5

A 29 year old woman complained of diffuse pain in the spine followed by difficulties in tasting and facial movements. At admission there was mild bilateral facial and deltoid weakness, with normal deep tendon reflexes. Blink reflex study disclosed an absence of all responses after stimulation of the supraorbital nerve on the right; left side stimulation evoked a delayed ipsilateral R2 with absent contralateral R2. The motor DL and the mean latency of $F$ wave of the right ulnar nerve were prolonged. The amplitude of CMAP of the ulnar and peroneal nerves of both sides was decreased. The CSF proteins were $1000 \mathrm{mg} / \mathrm{dl}$, with 2 cells $/ \mathrm{mm}^{3}$. Deep tendon reflexes disappeared in the days after admission.

When first seen the patient was already receiving steroids $(80 \mathrm{mg} /$ day methylprednisolone) which were gradually tapered; she subsequently received IVIg $(0.4 \mathrm{~g} / \mathrm{kg} /$ day for 5 days). The facial defect recovered within two weeks; diffuse pain was relieved by carbamazepine $(400 \mathrm{mg} /$ day $)$ given for a few days. At follow up, three months later, she was symptom free.

CASE 6

Ten days after a cholecystectomy this 52 year old woman complained of pain in the thighs (which increased on maintaining posture), paraesthesias (perioral and distally in all four limbs), and several falls caused by pain and hyposthenia of the legs.

Neurological evaluation showed lateral gaze nystagmus, severe hyposthenia in the lower limbs, and a mild loss of power in the right arm. Tendon reflexes were not elicitable in the four limbs and there was severe distal hypopallaesthesia.

Amplitudes of MCV and CAMP of both peroneal nerves were reduced, with an increased $\mathrm{F}$ wave latency, but conduction blocks were not seen. Sural nerve SAP was not elicitable in either side. Needle examination disclosed diffuse denervation with early signs of reinnervation. At CSF examination proteins were mildly increased $(64 \mathrm{mg} / \mathrm{dl})$ with 1 cell $/ \mathrm{mm}^{3}$.

This patient also underwent colonoscopy because of the appearance of diarrhoea and sensation of incomplete rectal emptying: a rectal ulceration was diagnosed and the biopsy showed suppurative inflammation. She was treated with plasma exchange (eight sessions) and subsequently received IVIg. Her neurological condition showed a slow and incomplete improvement and she was able to walk with a stick four months after onset. Further follow up was not available.

CASE 7

Three months before admission this 53 year old man experienced pain in the right forearm associated with difficulty in adducting and flexing the right arm; the pain progressively subsided, but the weakness persisted unchanged. In July 1993 he was admitted for sensory symptoms (short lasting "knife-like" pain and cold sensation) located in the cervical spine and the interscapular region, which were followed by diffuse hyposthenia, more pronounced in the distal upper limbs, and migrating pain in the shoulders and arms. Results of neurological examination were as follows: there was muscle power in the interossei, extensor, and flexor carpi ( $1 / 5$ on both sides); and deltoid, biceps brachii, and triceps (2-3/5), more pronounced on the left. A mild weakness was also present in the left peroneus longus, extensor hallucis longus, and extensor digitorum brevis. Triceps and right ankle reflexes were reduced; the other muscle stretch reflexes were normal.

Neurophysiological evaluation disclosed bilateral neurogenic morphological motor unit potential (MUP) changes in the $\mathrm{C} 7, \mathrm{C} 8$, and T1 territory; the amplitudes of CMAP (right ulnar and median nerves) and SAP (right median nerve) were markedly reduced. A cervical MRI was unremarkable. Examination of CSF showed an increased protein content $(1.42 \mathrm{~g} / \mathrm{dl})$ and $1 \mathrm{cell} / \mathrm{mm}^{3}$.

$\mathrm{He}$ was treated with plasma exchange (five sessions) and muscle strength substantially improved in the lower limbs, although a major power defect was still present in the upper limbs at discharge. When last seen, two years later, mild weakness (3/5) and muscle atrophy were present in both hands; muscle stretch reflexes had reappeared. A postural tremor was present in both hands.

\section{CASE 8}

A 62 year old man was admitted for paraesthesias in the hands and legs and fatiguability which had appeared 2 weeks earlier. One month before admission he had had bronchopneumonia. There was initially a mild distally predominant sensory (tactile) defect in the legs; power was normal. The ankle reflexes were absent. The CSF protein concentration was $63 \mathrm{mg} / \mathrm{dl}$, without cells. Ten days later hypopallaesthesia appeared in the distal legs, predominant on the right. Ankle reflexes were still absent, the other reflexes hypoactive. The MCV was reduced in the right median and 
peroneal nerves; sensory conduction velocity was reduced in the right median, right ulnar, and left sural nerves. The $F$ wave latency of the right median and right ulnar nerves and $\mathrm{H}$ reflex latency from the soleus were prolonged. He received steroids for 3 months; improvement began 5 weeks after the beginning of therapy. Recovery occurred after 3 months; 1 year later the patient, without therapy, had no symptoms.

CASE 9

This 58 year old woman was referred for neurological evaluation because of pain, pins and needles, and loss of power in the hands, which had appeared 2 weeks earlier. Symptoms gradually worsened and spread to the feet; she also complained of low back pain radiating to the legs. When first examined she presented bilateral ptosis, reduced power in the upper limbs (4/5 proximally, $2-3 / 5$ distally) and in the legs (4/5 iliopsoas, $3-4 / 5$ foot extensors). Atrophy of the first dorsal interosseous and thenar eminence of both hands was present. Medioplantar reflexes were absent, whereas other reflexes were slightly hyperactive. The CSF had $1 \mathrm{cell} / \mathrm{mm}^{3}$ and a protein concentration of 66 $\mathrm{mg} / \mathrm{dl}$. The SAP of sural nerves was not elicitable, and MCV of both peroneal nerves was reduced. Fibrillation potentials were found in the first dorsal interosseous of the right hand.

One week later power in the legs began to improve. The right knee reflex was absent and there was incomplete loss of tactile, pinprick, and vibration sense in the distal four limbs. A brain MRI disclosed some small hyperintense foci located within the periventricular white matter and the basal ganglia on both sides; a cervical MRI was normal. A search for lupus anticoagulant was positive.

She received steroids; functional recovery occurred one month after admission. At follow up, 1 year later, she was symptom free.

CASE 10

A woman, aged 73 , was treated with steroids until 1991 for polymyalgia rheumatica. In March 1992 she presented with a flu-like syndrome followed 10 days later by epigastric pain radiating on the right to the lumbar spine. The pain only appeared during the night. When first evaluated she presented superficial hypaesthesia in the legs (from the knees downwards) and in the abdomen (from D4-5 to L1), sensory ataxia, hypopallaesthesia, the absence of tendon reflexes in the lower limbs, and reduced muscle stretch reflexes in the arms. Power was $4 / 5$ in the triceps brachii and flexor digitorum of both sides, and 3/5 in the right iliopsoas; a mild weakness was present in the quadriceps of both sides and in the paraspinal muscles. Examination of CSF showed a protein concentration of $2110 \mathrm{mg} / \mathrm{dl}$ with 7 lymphocytes $/ \mathrm{mm}^{3}$.

Neurophysiological evaluation showed a severe axonal neuropathy predominating in the lower limbs, mostly involving the sensory fibres.

She received plasma exchange (six sessions). Six months later she had fully recovered from the motor defect, but hypopallaesthesia in the legs and sensory ataxia persisted.

CASE 11

Ten days before admission this 65 year old woman received intramuscular gangliosides for pain in the shoulders associated with headache and dizziness. She was admitted because of the sudden appearance of dysarthria, dysphonia, and dysphagia (which required enteral tube feeding). Three days later weakness appeared in the neck muscles, which extended to the upper limbs during the next day. Neurological evaluation also disclosed facial diplegia; the soft palate could not be raised on phonation, and the tongue could not be protruded. A severe weakness was found in the head extensors and flexors, scapular girdle, and upper limb muscles. The deep tendon reflexes were present, slightly brisk, and predominating on the left side in the arms. A bilateral Babinski's sign was also found. Hypotensive and hypertensive fits occurred during her stay in hospital.

A tensilon test was negative; cerebral and cervical MRI were unremarkable. Examination of CSF showed normal protein concentrations $(28 \mathrm{mg} / \mathrm{dl})$ with 8 cells $/ \mathrm{mm}^{3}$; one week later proteins rose to $51 \mathrm{mg} / \mathrm{dl}$ (without cells).

Denervation signs were found at EMG in the left triceps and biceps brachii; a reduced interference pattern was present in the left triceps, biceps brachii, and abductor pollicis brevis. Nerve conduction velocities were only mildly reduced in the left median nerve (MCV between elbow and wrist and third digit-wrist sensory conduction velocity), whereas normal findings were found in the left ulnar nerve. Both left median and ulnar nerves presented a markedly reduced CMAP amplitude with normal SAPs. A blink reflex study showed a prolonged R1 (+55\%) and ipsilateral and contralateral R2 ( $+20 \%)$ latency on both sides.

The patient received five sessions of plasma exchange as a first treatment and was subsequently treated with steroids. Her neurological condition gradually improved and she was discharged to home 2.5 months after admission. When last seen, 56 months later, there was still a mild bifacial weakness and soft palate mobility was reduced.

ANTIGANGLIOSIDE ANTIBODIES

All the patients, except for patient 5, were tested for antiganglioside antibodies as described above. Among the 10 tested patients only one (patient 7) was positive for IgG antibodies (anti-GM1) at time zero (at admission).

EXAMINATION OF CSF

The mean delay between onset of GuillainBarré syndrome and examination of CSF was 15.6 (SD 8.3) days. All the patients showed albuminocytological dissociation at some time during the course of the disease; however, there was a great variability in CSF protein concentration (mean (SD) 479 (708) $\mathrm{mg} / \mathrm{dl}$ ), and in $45 \%$ of patients $(5 / 11)$ the proteins were only mildly raised (49-66 mg/dl, table 3 ). Higher 
protein concentrations ( $\geqslant 1000 \mathrm{mg} / \mathrm{dl}$ ) were present in patients with axonal variants.

NEUROPHYSIOLOGICAL FINDINGS

An electrodiagnostic evaluation was performed in all cases; the mean time interval between onset of Guillain-Barré syndrome and neurophysiological examination was 15.9 (SD 9.3) days. The results were categorised as demyelinating in four patients $(36 \%)$ and axonal in five $(46 \%)$, whereas two patients $(18 \%)$ showed combined features (the corresponding figures for pooled Miller Fisher syndrome and cases fulfilling NINCDS criteria were $54 \%, 6 \%$, and $28 \%$, respectively).

\section{TREATMENT AND PROGNOSIS}

As first choice therapy, three patients (27\%) received IVIg and five $(46 \%)$ plasma exchange (mean number of sessions 5.4), whereas the remaining three $(27 \%)$ were treated with steroids. Overall, three patients $(27 \%)$ received a second cycle of therapy (two patients, first treated with plasma exchange, were subsequently given IVIg or steroids, one patient each; the third was taking steroids and subsequently received IVIg).

Relapses, defined according to Kleyweg and Van Der Mechè, ${ }^{10}$ were not found. Except for patient 6 , whose follow up only lasted 4 months, all the patients had recovered or were still alive 6 months after the onset of disease, and there were no deaths. Overall, recovery occurred in $6 / 11(55 \%)$ patients, whereas the other five showed improvement (in two of them major neurological signs remained). Recovery occurred in $4 / 4$ patients with demyelinating and in 1/5 with axonal findings on EMG .

\section{Discussion}

Incidence estimates are available for GuillainBarré syndrome cases fulfilling NINCDS criteria, the rates ranging between 1 and 2 cases/100 000 population/ year. ${ }^{81-17}$ For Guillain-Barré syndrome variants, defined diagnostic criteria have not been established as yet, and the frequency has usually been evaluated in clinical series, which could be subject to selection biases. In his series of 92 consecutive patients Ropper ${ }^{5}$ found that limited regional forms accounted for about $7 \%$ of those with Guillain-Barré syndrome. The rate was even higher in the series of Shuaib and Becker, the variants (excluding Miller Fisher syndrome) representing $13 \%$ of cases. ${ }^{18}$ In a recent paper by Beghi et al, ${ }^{7}$ which reported the results of a pilot study on the incidence of Guillain-Barré syndrome, the atypical cases (including pure motor Guillain-Barré syndrome, pure sensory Guillain-Barré syndrome, Miller Fisher syndrome, pharyngeal-cervical-brachial variant, paraparetic variant, and pure pandysautonomia) represented $6.4 \%$ of total cases; another $6.4 \%$ of cases, not fitting one of the above categories, could not be classified (details about these cases were not reported). Combining these "unusual" cases with "atypical" forms (excluding Miller Fisher syndrome) the calculated rate of Guillain-Barré syndrome variants was $8.2 \%$.
In our prospective study, which was based on a key informant system involving all the neurological clinics and departments within the study area, the incidence rate of GuillainBarré syndrome variants was $0.14 / 100000$ population/year (95\% CI 0.07-0.25), accounting for $10.5 \%$ of total cases of Guillain-Barré syndrome; this rate is comparable with those reported above. ${ }^{5} 718$

The analysis of clinical infectious antecedents during the month preceding onset of Guillain-Barré syndrome showed a lower frequency of upper respiratory tract infections for Guillain-Barré syndrome variants compared with the other forms of Guillain-Barré syndrome (pooled Miller Fisher syndrome and cases fulfilling NINCDS criteria), whereas influenza and gastrointestinal infections showed comparable frequencies (table 2). Before any conclusion can be drawn from such a finding it should be confirmed in further larger series, as our results are based on a few cases and therefore are to be viewed with caution.

All our incident cases presented as an acute polyneuropathy associated with albuminocytological dissociation which subsequently recovered; moreover, all cases were thoroughly evaluated for other possible aetiologies by extensive laboratory studies. Positivity for lupus anticoagulant was found in patient 9 , who, however lacked the clinical features of either systemic lupus erythematosus or primary antiphospholipid syndrome (recurrent venous and arterial thromboses, thrombocytopenia, fetal loss), ${ }^{19}$ thus suggesting that this finding represented an occasional positivity.

The analysis of clinical features disclosed two groups of patients. The first comprised cases with bifacial weakness associated with other signs (mainly sensory disturbances). These patients experienced a good outcome (almost always recovery or improvement with minor signs remaining); their electrophysiological findings were usually (but not always) categorised as demyelinating. The second group was clinically heterogeneous, including a case of pharyngeal-cervical-brachial variant and a pure sensory variant. One third of these patients recovered. Their EMG pattern was more often (but again not always) of an axonal type. As a whole $55 \%$ of our patients with Guillain-Barré syndrome variants showed cranial nerve involvement, the facial nerve being by far the most common, as previously reported. ${ }^{20}$

Because our study focused on the incidence, prognosis, and clinical features of GuillainBarré syndrome variants no treatment protocol was planned and each centre was free to decide about patients' therapy; for this reason and because of the few cases in our series we cannot draw any conclusion about the effect of therapy on prognosis of Guillain-Barré syndrome variants.

In conclusion, in our population-based study Guillain-Barré syndrome variants other than Miller Fisher syndrome accounted for $10 \%$ of all cases of Guillain-Barré syndrome and, as whole, they showed a good prognosis. Patients' 
clinical features were heterogeneous and facial diplegia, usually associated with other (sensory) signs, was the most frequent finding. The results of our study may be relevant for future epidemiological studies on Guillain-Barré syndrome.

This work was supported by a grant from the Ministero dell' Università e della Ricerca Scientifica e Technologica (MURST) quota $60 \%$.

\section{Appendix: Members of the Emilia- Romagna Group on Clinical and Epide- miological Problems in Neurology}

Principal investigators: $M$ Casmiro, $M$ Guarino, $R$ D’Alessandro. Participants: M Pasquinelli, B Currò Dossi (Department of Neurology, Rimini); W Neri, C Guidi, M, Gessaroli (Department of Neurology, Forli); F Rasi, R Fabbri, G Mazzini (Neurology Service, Cesena); GG Rebucci, G Padoan, C Callegarini, G Ciucci, AR Guidi (Department of Neurology, Ravenna); M Galeotti (Department of Neurology, Lugo); M Casmiro, G Bianchedi (Department of Neurology, Faenza); MR Tola, V Govoni, E Granieri (Institute of Neurology, University of Ferrara); G Tralli, V Tugnoli (Department of Neurology, Ferrara); V Mussuto (Neurology Service, Imola); M Guarino, G Azzimondi (Neurology Service, S Orsola-Malpighi Hospital, Bologna); A Baldrati, S Laudadio, T Sacquegna (Department of Neurology, Maggiore Hospital, Bologna); F Salvi (Department of Neurology, Bellaria Hospital, Bologna); G Ambrosetto (Institute of Neurology, University of Bologna), R D'Alessandro (Institute of Neurology, Neuroepidemiology Unit, University of Bologna); P Sorgato, V Ferrari, M Gentilini, A Colombo, E Merelli (Institute of Neurology, University of Modena); V Miele, V Lolli, M Santangelo (Department of Neurology, Carpi); L Motti, D Guidetti, F Solimè (Department of Neurology, Reggio Emilia); F Granella, G Pavesi, I Allegri, L Falco (Institute of Neurology, University of Parma); A Scaglioni, E Montanari (Department of Neurology, Fidenza); S Cammarata, V Poli (Department of Neurology, Piacenza). Immunological studies: A Lugaresi (Institute of Neurology, University of Chieti).

1 National Institute of Neurological and Communicative Disorders and Stroke Ad Hoc Committee: criteria for diagnosis of Guillain-Barré syndrome. Ann Neurol 1978;3:565-6.
2 Asbury AK. Diagnostic considerations in Guillain-Barré syndrome. Ann Neurol 1981;9(suppl):1-5.

3 Asbury AK, Cornblath DR. Assessment of current diagnostic criteria for Guillain-Barré syndrome. Ann Neurol 1990; 27(suppl):S21-4.

4 Arnason BGW, Soliven B. Acute inflammatory demyelinating polyradiculoneuropathy. In: Dyck PJ, Thomas PK, Griffin JW, et al, eds. Peripheral neuropathy. 3rd ed. Philadelphia: WB Saunders, 1993:1456-7.

5 Ropper AH. Unusual clinical variants and signs in GuillainBarré syndrome. Arch Neurol 1986;43:1150-2.

6 Ropper AH. Further regional variants of acute immune polyneuropathy. Bifacial weakness or sixth nerve paresis with paresthesias, lumbar polyradiculopathy, and ataxia with pharingeal-cervical-brachial weakness. Arch Neurol 1994;51:671-5.

7 Beghi E, Bogliun G for the Italian GBS Study Group. The Guillain-Barré syndrome (GBS). Implementation of a register of the disease on a nationwide basis. Ital $\mathcal{F}$ Neurol Sci 1996;17:355-61

8 Emilia-Romagna Study Group on Clinical and Epidemiological Problems in Neurology. A prospective study on the incidence and prognosis of Guillain-Barré syndrome in Emilia-Romagna region, Italy (1992-3). Neurology 1997; 48:214-21.

9 Lugaresi A, Ragno M, Torrieri F, et al. Acute motor axonal neuropathy with high titer IgG and IgA antiGD1a antibodies following Campylobacter enteritis. $\mathcal{F} \mathrm{Neurol} S \mathrm{Si}$ 1997;147:193-200.

10 Kleyweg RP, Van Der Mechè FGA. Treatment related fluctuations in Guillain-Barré syndrome after high-dose immunoglobulins or plasma-exchange. 7 Neurol Neurosurg Psychiatry 1991;54:957-60.

11 Alter M. The epidemiology of Guillain-Barré syndrome. Ann Neurol 1990;27(suppl):S7-12.

12 Beghi E, Kurland LT, Mulder DW, et al. Guillain-Barré syndrome. Clinicoepidemiologic features and effect of influenza vaccine. Arch Neurol 1985;42:1053-7.

13 Halls J, Bredkjær C, Friis ML. Guillain-Barré syndrome: diagnostic criteria, epidemiology, clinical course, and prognosis. Acta Neurol Scand 1988;78:118-22.

14 Koobatian TJ, Birkhead GS, Schramm MM, et al. The use of hospital discharge data for public health surveillance of Guillain-Barré syndrome. Ann Neurol 1991;30:618-21.

15 McLean M, Duclos P, Jacob P, et al. Incidence of GuillainBarré syndrome in Ontario and Quebec, 1983-9, using hospital service databases. Epidemiology 1994;5:443-8.

16 Paolino E, Govoni V, Tola MR, et al. Incidence of the Guillain-Barré syndrome in Ferrara, Northern Italy, 1981-7. Neuroepidemiology 1991;10:105-11.

17 Radhakrishnan K, El-Mangoush MA, Gerryo SE. Descriptive epidemiology of selected neuromuscular disorders in Benghazi, Libya. Acta Neurol Scand 1987;75:95-100.

18 Shuaib A, Becker WJ. Variants of Guillain-Barré syndrome: Miller Fisher syndrome, facial diplegia and multiple cranial nerve palsies. Can 7 Neurol Sci 1987;14:611-6.

19 Hess DC. Stroke associated with antiphospholipid syndrome. Stroke 1992;23 (suppl I):I-23-8.

20 Parry GJ. Guillain-Barré syndrome. New York: Thieme Medical, 1993. 